\title{
Gastric Adenocarcinoma at the Joliot Curie Institute in Dakar: Epidemiological, Diagnostic and Therapeutic Aspects about 54 Cases
}

\author{
Kanta Ka ${ }^{*}$, Doudou Diouf ${ }^{2,3}$, Sidy Ka ${ }^{2,3}$, El Hadj Amadou Sall' ${ }^{1}$, Daniele Presti ${ }^{4}$, \\ Mamadou Moustapha Dieng2,3, Papa Macoumba Gaye ${ }^{1,3}$, Ahmadou Dem ${ }^{2,3}$ \\ ${ }^{1}$ Radiotherapy Department, CHNU Dalal Jamm, Guédiawaye, Senegal \\ ${ }^{2}$ Oncology Department, Institute Joliot Curie, Dakar, Senegal \\ ${ }^{3}$ Cheikh Anta Diop University of Dakar, Dakar, Senegal \\ ${ }^{4}$ Medical Oncology Service, ICS Maugeri IRCCS SpA, University of Pavia, Pavia, Italy \\ Email: ^ka.kanta04@gmail.com, doudougaradiouf@gmail.com, sidy.ka@ucad.edu.sn, asall.jr@gmail.com, \\ daniele.presti91@gmail.com, adehdem@gmail.com, moustaphamamadou@gmail.com, macoumba.gaye@gmail.com
}

How to cite this paper: Ka, K., Diouf, D., Ka, S., Sall, ElH.A., Presti, D., Dieng, M.M., Gaye, P.M. and Dem, A. (2021) Gastric Adenocarcinoma at the Joliot Curie Institute in Dakar: Epidemiological, Diagnostic and Therapeutic Aspects about 54 Cases. Journal of Cancer Therapy, 12, 136-145. https://doi.org/10.4236/jct.2021.123015

Received: February 18, 2021

Accepted: March 22, 2021

Published: March 25, 2021

Copyright $\odot 2021$ by author(s) and Scientific Research Publishing Inc. This work is licensed under the Creative Commons Attribution-NonCommercial International License (CC BY-NC 4.0). http://creativecommons.org/licenses/by-nc/4.0/ (c) (i) (s) Open Access

\begin{abstract}
Objectives: To study the epidemiological, diagnostic, therapeutic and prognostic aspects of gastric adenocarcinoma at the Joliot Curie Institute in Dakar. Materials and Methods: We conducted a retrospective study over a seven-year period from January 2010 to December 2017 at the Joliot Curie Institute in Dakar. All cases of gastric adenocarcinoma proven by fibroscopy followed by histology or proven on the histological analysis of a surgical specimen were taken into account. The parameters studied were age, risk factors, stages of the disease, treatment and prognosis. Results: There were 54 cases of gastric adenocarcinoma over a period of 7 years. The average age was 54.74 years with extremes of 25 and 84 years. A male predominance was noted $(35 / 54)$. The main risk factors found were alcohol (5/54), tobacco (13/54), Helicobacter pilori (4/54), gastric ulcer (12/54). Epigastralgia was the most frequent clinical manifestation. FOGD was carried out in $77.8 \%$ of patients. Histology was obtained before surgery in 40 patients ( $74.1 \%$ of cases) and on the operating room in 14 patients (25.9\%). Patients were classified as stage II in $2 / 54$ cases, stage III in $5 / 54$ cases and stage IV in $47 / 54$ cases. Gastro-entero-anastomosis was the main surgical procedure performed. External radiotherapy was performed in 1/54 patients. Chemotherapy was done in $52 / 54$ patients, $96.3 \%$ of the cases. It was palliative in $66.7 \%$ of cases, neoadjuvant in $1.9 \%$ of cases, adjuvant in $24.1 \%$ of cases, perioperative in $3.7 \%$ of cases. Mortality was $79.6 \%$. Patient survival times were relatively short: in less than 6 months 24/54 cases, 13/54 cases between $6-12$ months, 5/54 cases between $13-24$ months and 6/54 cases beyond 24 months. 6/54 patients were
\end{abstract}


lost from view. Conclusion: Gastric adenocarcinoma is diagnosed lately in our conditions. It is responsible for a high mortality rate. Palliative treatment is often the only option because of the delay in diagnosis.

\section{Keywords}

Adenocarcinoma, Gastric, Survival

\section{Introduction}

Gastric cancer is the fifth most common cancer in the world and more than $70 \%$ of cases occur in developing countries. Adenocarcinoma is the most common histological type (90\%) [1].

In developing countries of gastric adenocarcinoma has decreased remarkably. This decrease in incidence is essentially due to a reduction in distal gastric cancers. This trend is largely explained by the regression of risk factors linked to the eradication of Helicobacter pylori and changes in dietary behavior [2].

Helicobacter pylori is the main risk factor for gastric adenocarcinoma [3].

In Senegal, the search for Helicobacter pylori is not systematic, sometimes because of limited diagnostic means or the cost fully covered by the patient.

The risk of gastric adenocarcinoma is increased in cases of atrophic gastritis and metaplasia, particularly when these lesions mainly involve the gastric body. The atrophic lesions may be the result of a Helicobacter pylori infection, Biermer's autoimmune gastritis or biliary reflux (partial gastrectomy). This risk has been estimated at $4 \%-6 \%$ according to old data from Correa et al. in patients with gastritis and metaplasia respectively [4].

Gastric cancer is associated with high salt consumption (the main factor) [5].

There is no organised screening policy in Senegal today, as there is in high-incidence countries such as Japan. Thus, the majority of these cancers are discovered at an advanced stage [3]. There are no specific clinical signs.

Since the advent of interventional endoscopy, the management of the early stages of the disease allows a survival rate of $95 \%$ at five years [6]. In contrast, the prognosis remains poor for patients who cannot undergo carcinological excisional surgery.

The aim of this work was to study the epidemiological, diagnostic, therapeutic and prognostic aspects of adenocarcinoma gastric cancer at the Joliot Curie Institute in Dakar.

\section{Materials and Methods}

We conducted a retrospective study over a seven-year period from January 2010 to December 2017 at the Joliot Curie Institute in Dakar.

\subsection{Patients}

All Gastric cancer files have been collected. 
All records of patients with a histological diagnosis of gastric adenocarcinoma were included. On the files selected, the criteria collected were age, sex, reason for consultation, risks factors, diagnostic means, treatment received, side effects, date of last consultation and status of the disease during follow-up.

Excluded were all records of patients whose histological diagnosis was not adenocarcinoma and all gastric adenocarcinoma lost after the first consultation.

\subsection{Treatment}

All patients received treatment either by surgery (curative/palliative) or chemotherapy (neoadjuvante/adjuvant) or radiation therapy.

\subsection{Follow up}

All patients were seen in consultation 1 month after treatment or contacted by telephone for those who did not show up for their appointment.

All patients who did not come for a follow-up consultation and who did not answer the phone on at least 10 calls regularly over 6 months were considered lost to follow-up.

\subsection{Statistical Analysis}

The categorical variables were calculated in mean, median, standard deviation.

Non-categorical variables: number and percentage.

Survival curves were calculated according to the Kaplan-Meier method.

\section{Results}

We had found 54 cases of gastric adenocarcinoma over a period of seven years, an average of 7 cases per year.

The average age was 54.74 years with extremes of 25 and 84 years. The 45 - 54 age group was the most represented [Figure 1]. There was a male predominance of $35 / 54$ with a sex ratio of 1.84 . The average age was 54.74 with extremes of 25 and 84.

The main risk factors found were alcohol (5/54), tobacco (13/54), Helicobacter pilori (4/54) and gastric ulcers (12/54). Epigastralgia (42/54) was the most frequent clinical manifestation, i.e. $77.8 \%$ of cases, followed by vomiting (22/54 cases). FOGD was performed in $77.8 \%$ of patients. Antral location was more frequent in 42/54 patients, followed by pyloric location in 28/54 patients and cardiac location in $9 / 54$ cases [Figure 2]. Histology was obtained before surgery in 40 patients, i.e. $74.1 \%$ of cases, and on the operating room in 14 patients (25.9\%). Thoraco-abdomino-pelvic CT scan with contrast injection was performed in all patients and revealed several sites of metastases in 37/54 cases [Table 1].

The patients were classified as stage II in 2/54 cases, stage III in 5/54 cases and stage IV in $47 / 54$ cases. Gastroentero-anastomosis was the main surgical procedure performed. External radiotherapy was performed in 1/54 patients. Chemotherapy was done in $52 / 54$ patients, i.e. $96.3 \%$ of the cases. It was palliative in 


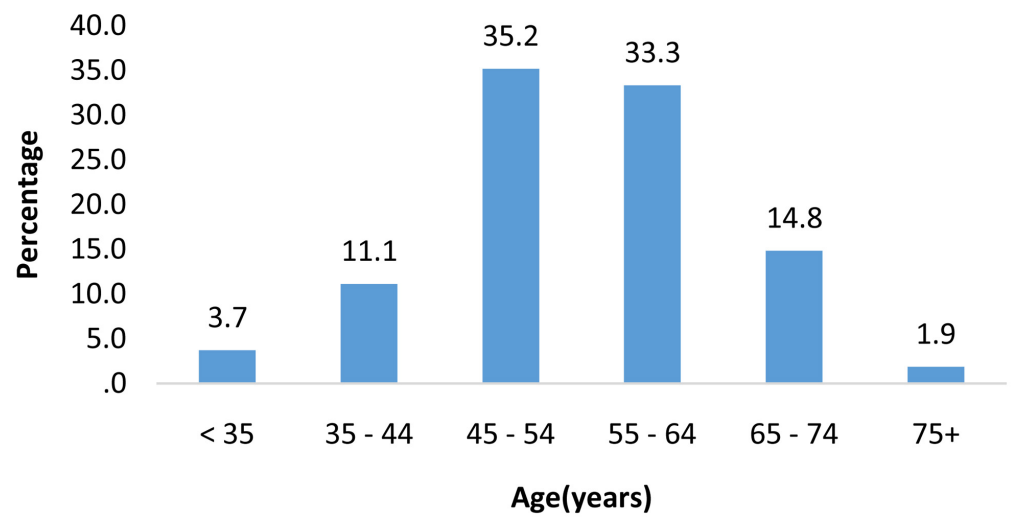

Figure 1. Breakdown of patients according to age.

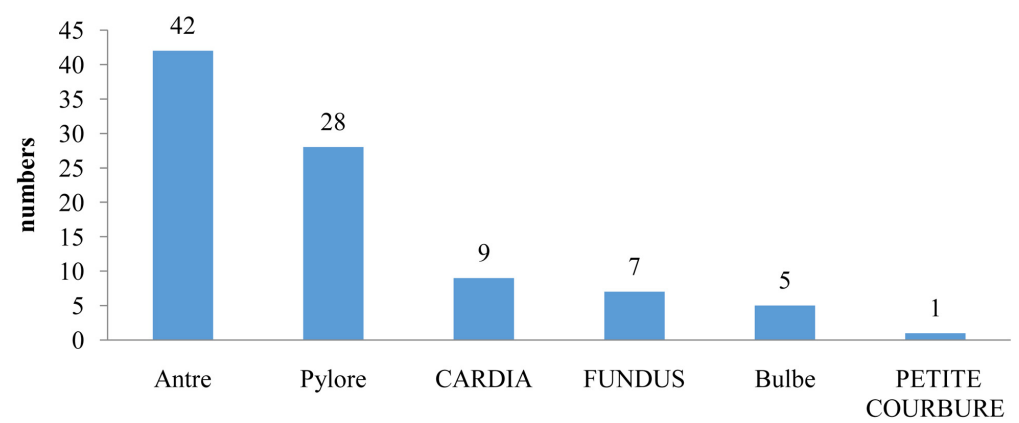

Figure 2. Partitioning of tumor seats at FOGD.

Table 1. Different sites of tumour metastasis on CT scan

\begin{tabular}{ccc}
\hline Sites of metastasis & Numbers & percentage (\%) \\
\hline Liver & 19 & 35.2 \\
Pancreas & 11 & 20.4 \\
lung & 8 & 14.8 \\
peritoneum & 6 & 11.1 \\
transverse colon & 2 & 3.7 \\
ovary & 1 & 1.9 \\
diaphragm & 1 & 1.9 \\
Duodenum & 1 & 1.9 \\
Node & 1 & 1.9 \\
round ligament & 1 & 1.9 \\
micronodule under pleural & 1 & 1.9 \\
hepatic adenopathy & 1 & 1.9 \\
kidney & 1 & 1.9 \\
Mesenteric vessels & 1 & 1.9 \\
\hline
\end{tabular}

$66.7 \%$ of cases, neoadjuvant in $1.9 \%$ of cases, adjuvant in $24.1 \%$ of cases, perioperative in $3.7 \%$ of cases. Mortality was $79.6 \%$ [Figure 3]. Patients had relatively short survival times: 24/54 cases within 6 months, 13/54 cases between 6 12 months, 5/54 cases between 13 - 24 months and 6/54 cases beyond 24 months 
[Table 2]. 6/54 patients were lost to follow-up.

\section{Discussion}

Several studies show that age over 60 is a factor linked to the risk of gastric adenocarcinoma, with a relative risk six to ten times higher [7].

In Morocco, the study by Ghizlane H. showed an average age of 56.66 years, with extremes ranging from 27 to 86 years, and the age of half of the patients (56\%) was between 50 and 70 years old [8]. In our study we have a younger population with a mean age of 54.74 years, with extremes of 25 and 84 years. The $68.5 \%$ were aged between 45 and 64, 14.8\% were under 45 and $16.7 \%$ were over 65.

In both developed and developing countries, the incidence is higher in men than in women [9]. In Senegal, the Faye I study. J. study estimated the sex ratio at 1.23 [10] slightly higher than the sex ratio in the IDRISSI S. study which was

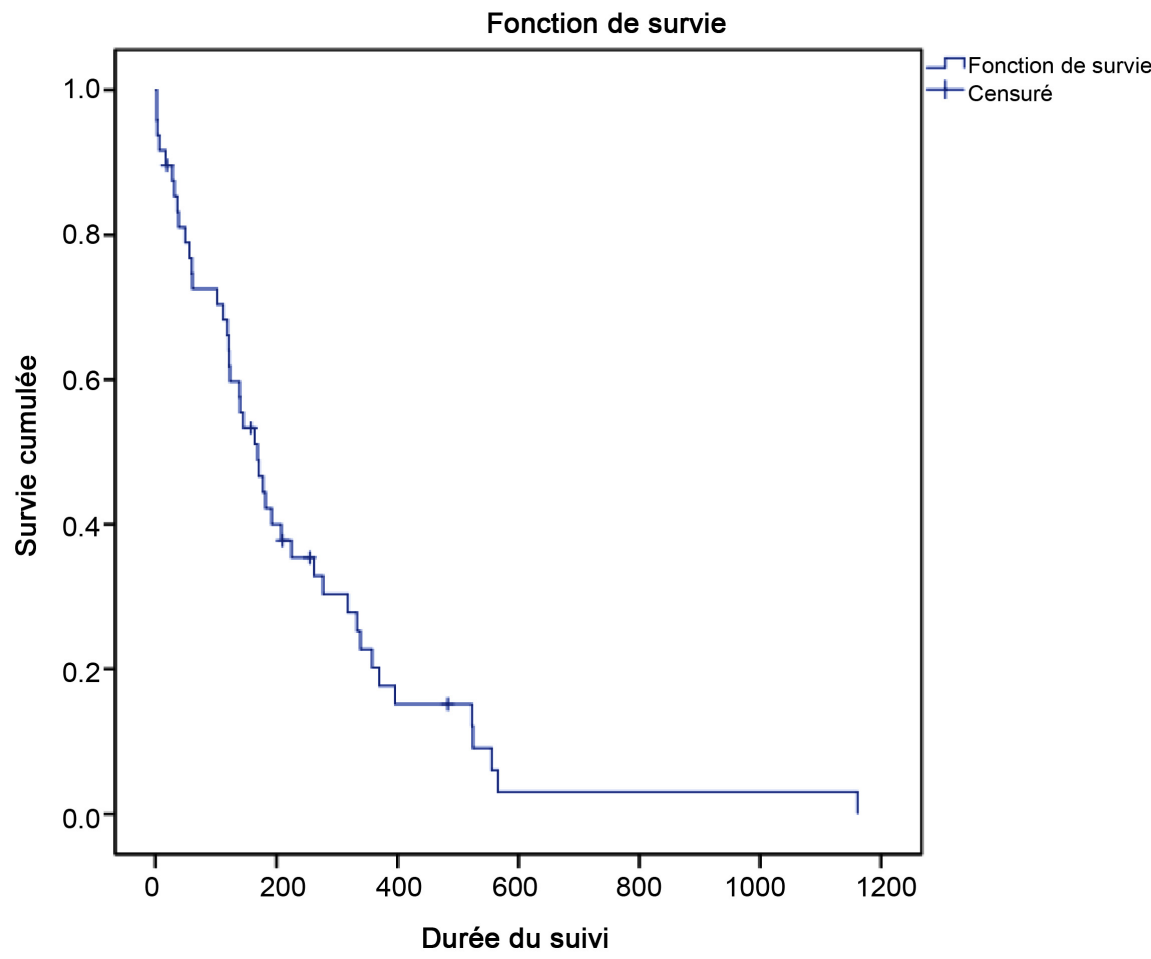

Figure 3. Overall patient survival according to Kaplan-Meier.

Table 2. Patient survival time.

\begin{tabular}{ccc}
\hline survival duration (month) & numbers & Percentage (\%) \\
\hline$<6$ & 24 & 50.0 \\
$6-12$ & 13 & 27.1 \\
$13-24$ & 5 & 10.4 \\
$>24$ & 6 & 12.5 \\
Total & 48 & 100 \\
\hline
\end{tabular}


1.125 [11]. In our series the sex ratio was estimated to be 1.84; a higher figure than the two above-mentioned studies with still a male predominance.

Ladeiras-Lopes $\mathrm{R}$ et al. examined the relationship between smoking and gastric adenocarcinoma. A meta-analysis of 42 studies estimated that the risk was increased by about 1.53 times and was higher in men [12]. A prospective study in Europe (EPIC) found a similar magnitude of risk, which decreased after 10 years of smoking cessation. About $18 \%$ of gastric cancer cases were attributed to smoking [13]. In our study, 13 patients were tobacco users, i.e. $24.1 \%$.

Moreover, for alcohol, a constant association between alcohol consumption and the risk of gastric cancer has not been demonstrated [14] [15]. A European study has suggested that daily wine intake could be protective [14].

We noted 5 cases, i.e. 9.3\% of occasional alcohol consumption, this figure being not significant for establishing a relationship with gastric adenocarcinoma.

The International Agency for Research on Cancer of the World Health Organisation classifies $H$. pylori as a group 1 or defined carcinogen [16]. Only distal adenocarcinoma and not adenocarcinoma of the cardia was linked to $\mathrm{H}$. pylori infection. A Swedish case-control study comparing 298 subjects with gastric cancer to 244 controls showed that more than $70 \%$ of distal gastric cancers were linked to H. pylori. In 2001, Uemura et al. reported a large prospective Japanese study involving 1528 subjects followed for an average of 7.8 years by endoscopy every one to three years. Thirty-six subjects had cancer during the follow-up. All cancers occurred among the 1246 infected patients and no cancer was found among the 280 uninfected. According to our data, the bacterium was detected in 13 patients, i.e. $24.1 \%$.

Epigastralgia and vomiting were the most frequent signs found as functional signs in our study; in $77.8 \%$ and $40.8 \%$ of cases respectively. The same signs were found in the study by Idrissi S. [11].

Oeso-gastro-duodenal fibroscopy should be requested as a first-line treatment, which, combined with biopsies, allows diagnosis in 95\% of cases. Endoscopy makes it possible to assess the size, location and extent of the tumour [17]. Cancers of the antrum are the most common (60\%) [17]. In their study, Kadend P et al. found $59.6 \%$ of the tumour had an anteropyloric site [13], while Idrissi S's study also showed a predominance of the anteropyloric site [11], as did Ghizlane H., which had a percentage of $65.5 \%$ [8]. In our study, the anteropyloric seat was predominant, as in the above-mentioned series, with $77.8 \%$ in the den and $51.85 \%$ in the pylorus. The cardiac seat was not negligible with a percentage of $16.7 \%$.

The helical abdominal pelvic scanner is the initial stage of the extension assessment. However, this examination tends to underestimate locoregional extension. According to the series, the concordance with tumor-nodes-metastases (TNM) is for $\mathrm{T}$ from $51 \%$ to $67 \%$, for $\mathrm{N}$ from $51 \%$ to $73 \%$, for hepatic metastases from $57 \%$ to $87 \%$ and for peritoneal carcinosis from $30 \%$ to $71 \%$. In the series of Ghizlane H. Hepatic and pulmonary metastases and peritoneal carcinosis were the most frequent metastatic localisations found in $9.5 \%, 8.6 \%$ and $9.5 \%$ of cases 
respectively. As in our case, liver metastases were predominant at $35.19 \%$, followed by pancreatic metastases at $20.37 \%$, pulmonary metastases at $14.81 \%$ and peritoneal metastases at $11.11 \%$ [Table 1].

After an extension assessment that revealed no metastases, complete surgical resection of the primary tumour and regional adenopathies is the only curative option for gastric cancers. Palliative resection may be indicated in the case of a symptomatic stenosing or haemorrhagic tumour. If the tumour cannot be removed, a gastroenterostomy can be performed in the case of a stenosing distal tumour or a prosthesis can be inserted [18].

Among our patients $37.04 \%$ had benefited from a GEA, in Morocco Ghizlane H. reports $29.4 \%$ palliative surgery [13], while in Japan a study found $0 \%$ palliative surgery [19]. This can be explained by the early detection organised in countries with a high incidence of gastric adenocarcinoma, and in our country the disease is discovered at an advanced stage.

In 2014, Xiong et al. published a meta-analysis based on the results extracted from published trial reports on 1820 patients from 12 different studies. Of these 12 studies, six were from Asia and six were from Western countries. The median follow-up period was 53 months. The meta-analysis showed that patients treated with neoadjuvant chemotherapy plus surgery had only a slightly improved survival benefit compared to patients treated with surgery alone. However, the 3 -year progression-free survival rate, tumour staging rate and $\mathrm{R} 0$ resection rate were better in patients treated with neoadjuvant chemotherapy plus surgery [20]. In our series 1 patient received neoadjuvant chemotherapy. We note that neoadjuvant chemotherapy is not commonly adopted. And this result does not allow us to know the impact of neoadjuvant chemotherapy.

Over the past two decades, six meta-analyses have been published on the role of adjuvant chemotherapy in gastric adenocarcinoma [21] [22].

Five of these six meta-analyses found improved survival after adjuvant chemotherapy compared to surgery alone [23] [24].

13 of our patients had adjuvant chemotherapy, i.e. $24.1 \%$. Of the 13 patients, $76.9 \%$ had a progression of gastric cancer, $15.4 \%$ had a partial response and $7.7 \%$ a complete response. With the low socio-economic level there is a problem of treatment compliance (number of cures not respected), which explains the results obtained. However, the percentage of complete response is not negligible, which shows the beneficial effect of the adjuvant treatment.

Two randomised trials in Western countries have evaluated perioperative chemotherapy in gastric cancer. The United Kingdom Medical Research Council's randomized trial of intravenous infusion chemotherapy for adjuvant gastric cancer (MAGIC) compared surgery with or without perioperative ECF chemotherapy (epirubicin, cisplatin, perfused fluorouracil). In this study, approximately $25 \%$ and $50 \%$ of patients were treated for gastric cancer and received D1 or D2 surgery, respectively. Of the $86 \%$ of patients assigned to perioperative chemotherapy who received preoperative chemotherapy, only 55\% also received postoperative chemotherapy. In this study, perioperative chemotherapy improved 
overall survival and local and remote control compared to surgery alone [25]. In our series we had 2 patients with perioperative chemotherapy. In contrast to the studies cited above, our patients had a progression of the cancer.

Chemotherapy is the reference treatment for gastric adenocarcinoma in locoregional and metastatic recurrence. The impact of these chemotherapies in terms of survival remains modest. The main objective of these palliative treatments is still local symptom control in order to improve patients' quality of life [25]. In our series, 36 patients or $66.7 \%$ had palliative chemotherapy: $75 \%$ had an unfavourable response, $8.33 \%$ had a stationary disease and $16.67 \%$ had a partial response.

We have a significant number of palliative chemotherapy patients because of the delay in consultation and diagnosis at the advanced stage of the disease.

There has been a steady decline in mortality from gastric adenocarcinoma. A study of gastric adenocarcinoma mortality in Europe and other parts of the world between 1980 and 2005 showed an annual percentage change in the gastric mortality rate of around $3-4$ per cent for the major European countries [26]. Patients with resectable gastric adenocarcinoma have a poor prognosis with an overall 5-year survival rate of about $20 \%$ to $30 \%$ worldwide [27]. Ghizlane $\mathrm{H}$. had an overall survival rate of $79.3 \%$ at 6 months and $5.2 \%$ at 5 years [13]. In our study we had $79.6 \%$ dead patients, $9.26 \%$ living patients and $11.1 \%$ lost to follow-up. This confirms that gastric adenocarcinoma has a poor prognosis if treatment is late and especially if there is a delay in diagnosis. The majority of

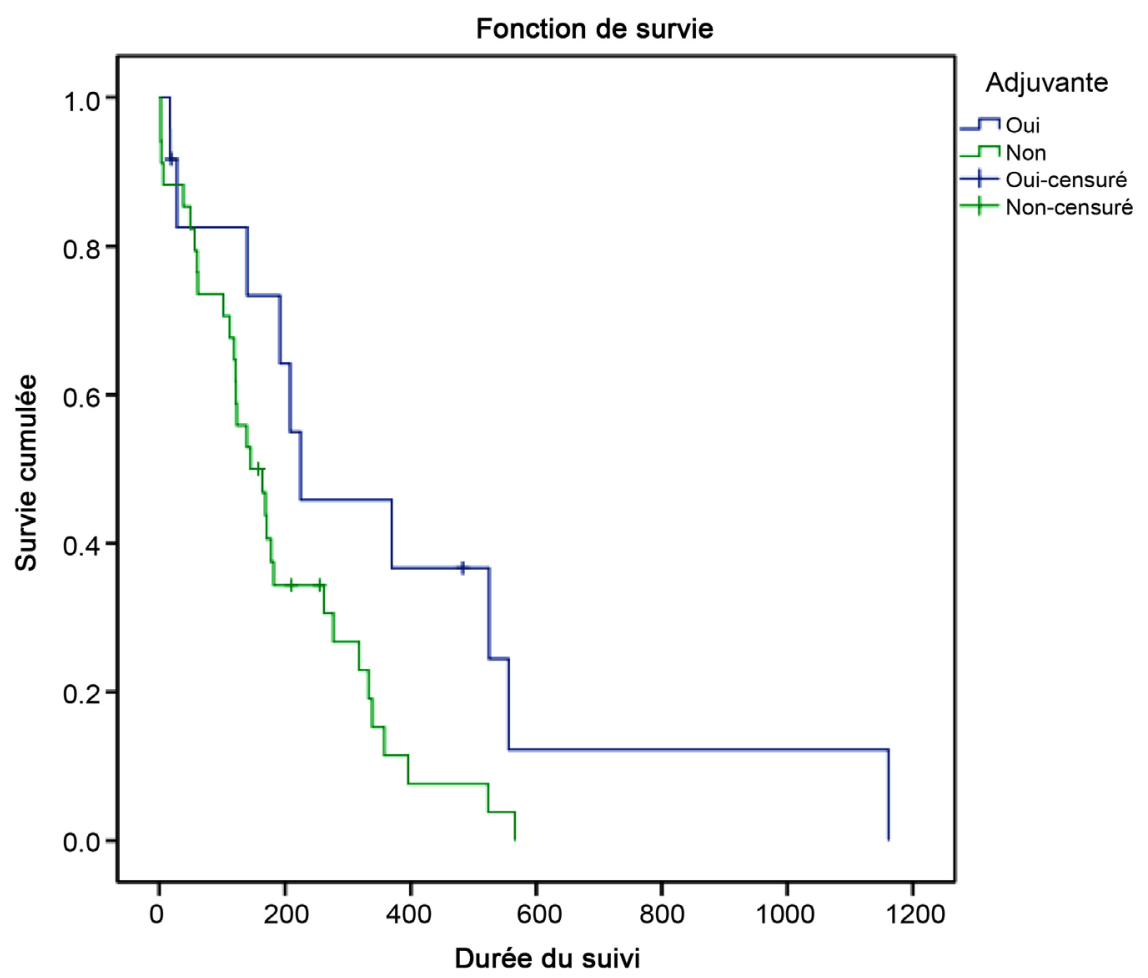

Figure 4. Survival curve of patients who received adjuvant chemotherapy compared to the survival curve of other types of chemotherapies. 
patients consulted at a terminal stage, which explains the predominant palliative chemotherapy with a poor survival rate. Furthermore, we note a better survival rate in patients who had adjuvant chemotherapy [Figure 4].

\section{Conclusion}

Gastric adenocarcinoma is less common in sub-Saharan Africa. Its evolution is deaf explaining the diagnosis at metastatic stages. It is a cancer with a poor prognosis. Improving access to quality care and raising public awareness will reduce mortality.

\section{Conflicts of Interest}

The authors declare no conflicts of interest regarding the publication of this paper.

\section{References}

[1] Aparicot, T., Yacoub, M., Karila-Cohen, P., et al. (2004) Adénocarcinome gastrique: Notions fondamentales, diagnostic et traitement. EMC-Chirurgie, 1, 47-66. https://doi.org/10.1016/j.emcchi.2003.10.003

[2] Richard, N., et al. (2019) Cancers de l'estomac. Traité de medicine AKOS, 2, 1-7.

[3] Gangloff, A. and Michel, P. (2013) Cancers de l'estomac. EMC-AKOS (Traité de Médecine), 8, 1-6.

[4] Correa, P., Haenszel, W., Cuello, C., Zavala, D., Fontham, E., Zarama, G., et al. (1990) Gastric Precancerous Process in a High Risk Population: Cohort Follow-Up. Cancer Research, 50, 4737-4740.

[5] D’Elia, L., Rossi, G., Ippolito, R., Cappuccio, F.P. and Strazzullo, P. (2012) Habitual Salt Intake and Risk of Gastric Cancer: A Meta-Analysis of Prospective Studies. Clinical Nutrition, 31, 489-498. https://doi.org/10.1016/j.clnu.2012.01.003

[6] Ajani, J.A., Lee, J., Sano, T., Janjigian, Y.Y., Fan, D. and Song, S. (2017) Gastric Adenocarcinoma. Nature Reviews Disease Primers, 3, Article No. 17036. https://doi.org/10.1038/nrdp.2017.36

[7] Watabe, H., Mitsushima, T., Yamaji, Y., et al. (2005) Prévision du développement du cancer gastrique à partir de la combinaison des anticorps d'Helicobacter pylori et du statut des pepsinogènes sériques: Une étude de cohorte endoscopique prospective. Gut, 54, 764-768. https://doi.org/10.1136/gut.2004.055400

[8] Ghizlane, H. (2016) L’adénocarcinome gastrique: Etude analytique. Thèse de médecine FES N051/16.

[9] Lambert, R. (2010) Épidémiologie du cancer gastrique dans le monde. Cancéro digest, 2, 31-37. https://doi.org/10.4267/2042/30746

[10] Faye, I.J. (2010) Prise en charge des cancers gastriques au service de chirurgie générale de l'hôpital Grand Yoff. Thèse de médecine, Dakar, $\mathrm{N}^{\circ} 75$.

[11] Idrissi, S. (2013) Les cancers de l'estomac à l'institut Juliot curie de l'hôpital Aristide Le Dantec de Dakar à propos de 136 cas. Thèse de médecine, UCAD, $\mathrm{N}^{\circ} 132$.

[12] Ladeiras-Lopes, R., Periera, A.K., Nogueira, A., et al. (2008) Smoking and Gastric Cancer: Systematic Review and Meta-Analysis of Cohort Studies. Cancer Causes Control, 19, 689. https://doi.org/10.1007/s10552-008-9132-y

[13] Gpnzalez, C.A., Pera, G., Agudo, A., et al. (2003) Smoking and the Risk of Gastric 
Cancer in the European Prospective Investigation into Cancer and Nutrition (EPIC). International Journal of Cancer, 107, 629-634.

https://doi.org/10.1002/ijc.11426

[14] Barstad, B., Sorensen, T., Tjonneland, A., et al. (2005) Intake of Wine, Beer and Spirits and Risk of Gastric Cancer. European Journal of Cancer Prevention, 14, 239. https://doi.org/10.1097/00008469-200506000-00007

[15] Tramacere, I., Negri, E., Pelucchi, et al. (2012) Une méta-analyse sur la consommation d'alcool et le risque de cancer gastrique. Annals of Oncology, 23, 28. https://doi.org/10.1093/annonc/mdr135

[16] (1994) Groupe de travail du CIRC sur l'évaluation des risques cancérogènes pour l'homme, les schistosomes, les douvements hépatiques et Helicobacter pylori. Vol. 61 des monographies du CIRC sur l'évaluation des risques cancérogènes pour l'homme. Centre international de recherche sur le cancer, Lyon, Vol. 61.

[17] Karila-Cohen, P., Petit, T., Aparicio, T., et al. (2004) Adénocarcinome gastrique Éditions Françaises de Radiologie, Paris, 2004. Journal de Radiologie, 85, 1695-1698. https://doi.org/10.1016/S0221-0363(04)97733-5

[18] Aparicot, T., Yacoub, M., Karila-Cohen, P., et al. (2004) Gastric Carcinoma: Epidemiology, Diagnosis and Treatment. EMC-Chirurgie, 1, 47-66. https://doi.org/10.1016/j.emcchi.2003.10.003

[19] Takemoto, Y. (2003) Caractéristiques clinicopathologiques du cancer gastrique précoce: Résultats de 100 cas d'un hôpital général rural. The European Journal of Surgery, 165, 319-325.

[20] Xiong, B., Ma, L., Cheng, Y., et al. (2014) Clinical Effectiveness of Neoadjuvant Chemotherapy in Advanced Gastric Cancer: An Updated Meta-Analysis of Randomized Controlled Trials. European Journal of Surgical Oncology, 40, 1321-1330. https://doi.org/10.1016/j.ejso.2014.01.006

[21] Hermans, J., Bonenkamp, J.J. and Boon, M.C. (1993) Traitement adjuvant après une résection curative pour le cancer gastrique: Méta-analyse d'essais randomisés. Journal of Clinical Oncology, 11, 1441-1447. https://doi.org/10.1200/JCO.1993.11.8.1441

[22] Paoletti, X., Oba, K., Burzykowsky, T., et al. (2010) Benefit of Adjuvant Chemotherapy for Resectable Gastric Cancer: A Meta-Analysis. JAMA, 303, 1729-1737.

https://doi.org/10.1001/jama.2010.534

[23] Earle, C.C. and Maroun, J.A. (1999) Adjuvant Chemotherapy after Curative Resection for Gastric Cancer in Non-Asian Patients: Revisiting a Meta-Analysis of Randomised Trials. European Journal of Cancer, 35, 1059-1064. https://doi.org/10.1016/S0959-8049(99)00076-3

[24] Cunningham, D., Allum, W., Stenning, S.P., et al. (2006) Perioperative Chemotherapy versus Surgery Alone for Resectable Gastroesophageal Cancer. The New England Journal of Medicine, 355, 11. https://doi.org/10.1056/NEJMoa055531

[25] Mornex, F. and Chauffert, B. (1998) Chimioradiothérapie concomitante dans la stratégie thérapeutique des adénocarcinomes du pancréas exocrine et de l'estomac. Cancerl Radiothérapie, 2, 696-702. https://doi.org/10.1016/S1278-3218(99)80010-9

[26] Bertuccio, P., Chatenoud, L., Levi, F., et al. (2009) Recent Patterns in Gastric Cancer: A Global Overview. International Journal of Cancer, 125, 666-673. https://doi.org/10.1002/ijc.24290

[27] Quero, L., Guillerm, S. and Hennequin, C. (2015) Néoadjuvant ou thérapie adjuvante pour le cancer gastrique. World Journal of Gastrointestinal Oncology, 7, 102-110. 